\title{
Algorithmic correspondence for intuitionistic modal mu-calculus, Part 2 (Extended Abstract)
}

\author{
Willem Conradie ${ }^{1}$, Yves Fomatati ${ }^{2}$, Alessandra Palmigiano ${ }^{2}$, and Sumit Sourabh ${ }^{2}$ \\ 1 University of Johannesburg, South Africa \\ wconradie@uj.ac.za \\ 2 ILLC, University of Amsterdam, The Netherlands \\ A.Palmigiano@uva.nl, S.Sourabh@uva.nl
}

\section{Introduction}

Sahlqvist-style correspondence results remain a perennial theme and an active topic of research within modal logic. Recently, there has been interest in extending the classical results in this area to the modal mu-calculus [7]. For instance, in [8] van Benthem, Bezhanishvili and Hodkinson define a class of Sahlqvist formulas for the modal mu-calculus, all of which have frame correspondents in first-order logic with least fixed points (FO+LFP). The analysis pursued in that paper is model theoretic in the tradition of the well known Sahlqvist-van Benthem algorithm.

Parallel to the model theoretic approach to this type of result, there exists an algebraic-algorithmic approach (see e.g., [3, 4, 5]) which derives correspondence (and canonicity) results by means of 'calculi of correspondence' consisting of simple derivation rules which depend for their soundness on the order theoretic properties of the operations interpreting the logical connectives in the algebraic semantics. As indicated in Part 1 [2], these rules are divided into approximation and adjunction rules, together with the Ackermann rules used to eliminate propositional variables. As also indicated in [2], the major challenge to extending the algebraic-algorithmic approach to the modal mu-calculus is obtaining sound approximation and adjunction rules for formulas involving fixed point binders: in the binder-free setting each connective can be treated in isolation on its own order-theoretic merits; in the presence of binders the the analysis must take into account the order-theoretic behaviour of whole formulas. As a result the derivation rules become significantly more involved.

Outline. In this talk we shall:

1. show how the calculus of correspondence, and hence the ALBA algorithm, can be extended to handle the intuitionistic modal mu-calculus, by adding the recursive Ackermann-rules and the residuation and adjunction rules for $\mu$-formulas discussed in [2];

2. define the class of recursive $\mu$-inequalities and compare it with related classes in the literature;

3. show that the extended ALBA successfully eliminates all propositional variables from all recursive $\mu$-inequalities;

4. conclude that, consequently, all recursive $\mu$-inequalities have frame correspondents in first-order logic with least fixed points. 
Language and semantics. We will be working in the language $\mathcal{L}$ of intuitionistic modal logic augmented with the connective -. This language will be interpreted on modal bi-Heyting algebras: an algebra $(A, \wedge, \vee, \rightarrow,-, \top, \perp)$ such that both $(A, \wedge, \vee, \rightarrow, \top, \perp)$ and $(A, \wedge, \vee,-, \top, \perp)^{\partial}$ are Heyting algebras is a bi-Heyting algebra; a modal bi-Heyting algebra is an algebra $(A, \wedge, \vee, \rightarrow,-, \top, \perp, \square, \diamond)$ such that $(A, \wedge, \vee, \rightarrow,-, \top, \perp)$ is bi-Heyting algebra and $\square$ and $\diamond$ preserve finite meets and joins, respectively, and $\square T=T$ and $\diamond \perp=\perp$. The relational duals of these algebras are intuitionistic modal Kripke frames, i.e., the usual intuitionistic Kripke frames augmented with (constrained) relations for interpreting $\square$ and $\diamond$. The language $\mathcal{L}^{+}$extends $\mathcal{L}$ with the addition of the connectives $\diamond$ and $\boldsymbol{\square}$ interpreted in perfect bi-Heyting algebras as the left and right adjoints of $\square$ and $\diamond$ respectively, and with special variables $\mathrm{NOM}=\{\mathbf{j}, \mathbf{i}, \mathbf{k}, \ldots\}$ and $\mathrm{CNOM}=\{\mathbf{m}, \mathbf{n}, \mathbf{l}, \ldots\}$ which range over the sets $J^{\infty}(\mathbb{C})$ and $M^{\infty}(\mathbb{C})$ of join- and meet-irreducible elements of perfect bi-Heyting algebras $\mathbb{C}$.

\section{The calculus of correspondence and the algorithm ALBA}

The calculus of correspondence consists of rewrite rules applicable to sets of inequalities in the language $\mathcal{L}^{+}$. The most important rule is the recursive Ackermann rule, based on a recursive version of Ackermann's Lemma:

$$
\frac{\exists p\left[\&_{i=1}^{n} \alpha_{i}(p) \leq p \& \&_{j=1}^{m} \beta_{j}(p) \leq \gamma_{j}(p)\right]}{\&_{j=1}^{m} \beta_{j}\left(\mu p \cdot\left[\bigvee_{i=1}^{n} \alpha_{i}(p)\right] / p\right) \leq \gamma_{j}\left(\mu p \cdot\left[\bigvee_{i=1}^{n} \alpha_{i}(p)\right] / p\right)}\left(\mathrm{RA}_{r e c}\right)
$$

subject to the restrictions that the $\alpha_{i}(p)$ and $\beta_{j}$ are positive in $p$ and that the $\gamma_{j}$ are negative in $p$.

Applied from top to bottom, this rule eliminates the propositional variable $p$. Since it is the presence of propositional variables which is responsible for the second-order quantification in the standard frame-correspondents, it is the aim of the ALBA algorithm (as an 'implementation' of the calculus of correspondence) to eliminate all occurring propositional variables from a given $\mathcal{L}$-inequality. Through the approximation and adjunction rules (discussed in part $1[2]$ ) inequalities are brought into a form to which $\left(\mathrm{RA}_{r e c}\right)$, or an order-dual version of it, is applicable. This comes at the cost of introducing syntax from the extended language $\mathcal{L}^{+}$. This is harmless, as the standard first-order translation on intuitionistic modal Kripke frames extends to accommodate the adjoints $\bullet$ and $\mathbf{m}$, and moreover the join- and meet-irreducible elements of perfect bi-Heyting algebras over which the special variables in NOM and CNOM range correspond to first-order definable subsets of these frames.

For a complete list of all the rules of deduction employed by ALBA, please refer to [1].

\section{The recursive $\mu$-inequalities}

In this section we include in the $\mathcal{L}$ the binary connectives $\circ$ and $\star$, the algebraic interpretations of which are,respectively, $\vee$-preserving and $\wedge$-preserving in both coordinates. This is done to widen the applicability of the of the ensuing definitions to a broader spectrum of logics.

An order-type over $n \in \mathbb{N}$ is an $n$-tuple $\epsilon \in\{1, \partial\}^{n}$. For every order-type $\epsilon$, let $\epsilon^{\partial}$ be its opposite order-type, i.e., $\epsilon_{i}^{\partial}=1$ iff $\epsilon_{i}=\partial$ for every $1 \leq i \leq n$.

For any $\mathcal{L}$-sentence $\varphi\left(p_{1}, \ldots p_{n}\right)$, any order-type $\epsilon$ over $n$, and any $1 \leq i \leq n$, an $\epsilon$-critical node in the signed generation tree of $\varphi$ is a (leaf) node $+p_{i}$ with $\epsilon_{i}=1$, or $-p_{i}$ with $\epsilon_{i}=\partial$. An $\epsilon$-critical branch in the tree is a branch terminating in an $\epsilon$-critical node. The intuition, which will be built upon later, is that variable occurrences corresponding to $\epsilon$-critical nodes are to be solved for, according to $\epsilon$.

For every $\mathcal{L}$-sentence $\varphi\left(p_{1}, \ldots p_{n}\right)$, and every order-type $\epsilon$, we say that $+\varphi$ (resp. $-\varphi$ ) agrees with $\epsilon$, and write $\epsilon(+\varphi)$ (resp. $\epsilon(-\varphi)$ ), if every leaf node in the signed generation tree of $+\varphi$ (resp. $-\varphi$ ) 


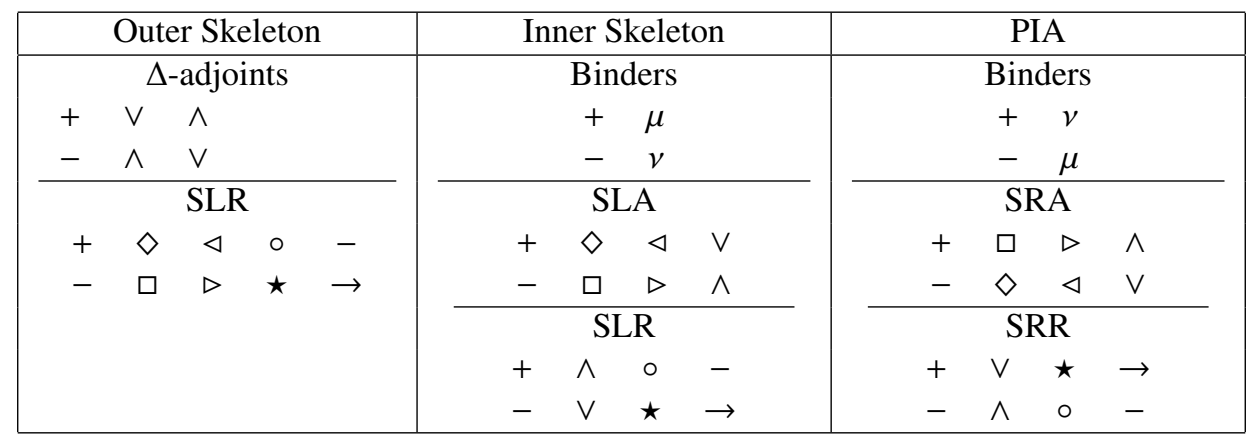

Table 1: Skeleton and PIA nodes.

which is labelled with a propositional variable is $\epsilon$-critical. In other words, $\epsilon(+\varphi)$ (resp. $\epsilon(-\varphi)$ ) means that all propositional variable occurrences corresponding to leaves of $+\varphi$ (resp. $-\varphi$ ) are to be solved for according to $\epsilon$. We will also make use of the sub-tree relation $\gamma<\varphi$, which extends to signed generation trees, and we will write $\epsilon(\gamma) \prec * \varphi$ to indicate that $\gamma$, regarded as a sub- (signed generation) tree of $* \varphi$, agrees with $\epsilon$.

Definition 3.1. Nodes in signed generation trees will be called skeleton nodes and PIA nodes according to the specification given in table 1 . A branch in a signed generation tree $* \varphi$, for $* \in\{+,-\}$, ending in a propositional variable is an $\epsilon$-good branch if, apart from the leaf, it is the concatenation of three paths $P_{1}, P_{2}$, and $P_{3}$, each of which may possibly be of length 0 , such that $P_{1}$ is a path from the leaf consisting only of PIA-nodes, $P_{2}$ consists only of inner skeleton-nodes, and $P_{3}$ consists only of outer skeleton-nodes. Moreover,

1. The formula corresponding to the uppermost node on $P_{1}$ is a mu-sentence.

2. On any SRR-node in $P_{1}$ of the form $\gamma \odot \beta$, where $\beta$ is the side where the branch lies, $\gamma$ is a musentence and $\epsilon^{\partial}(\gamma) \prec * \varphi$ (see above for this notation).

Unpacking the condition $\epsilon^{\partial}(\gamma) \prec * \varphi$ specifically to the $\mathcal{L}$-signature, we obtain:

a) if $\gamma \odot \beta$ is $+(\gamma \star \beta),+(\gamma \vee \beta),+(\beta \rightarrow \gamma)$, or $-(\beta-\gamma)$, then $\epsilon^{\partial}(+\gamma)$;

b) if $\gamma \odot \beta$ is $+(\gamma \rightarrow \beta),-(\gamma \wedge \beta),-(\gamma \circ \beta)$, or $-(\gamma-\beta)$, then $\epsilon(+\gamma)$.

3. On any SLR-node in $P_{2}$ of the form $\gamma \odot \beta$, where $\beta$ is the side where the branch lies, $\gamma$ is a musentence and $\epsilon^{\partial}(\gamma) \prec * \varphi$ (see above for this notation).

Unpacking the condition $\epsilon^{\partial}(\gamma) \prec * \varphi$ specifically to the $\mathcal{L}$-signature, we obtain:

a) if $\gamma \odot \beta$ is $-(\gamma \star \beta),-(\gamma \vee \beta),-(\beta \rightarrow \gamma)$, or $+(\beta-\gamma)$, then $\epsilon(+\gamma)$;

b) if $\gamma \odot \beta$ is $-(\gamma \rightarrow \beta),+(\gamma \wedge \beta),+(\gamma \circ \beta)$, or $+(\gamma-\beta)$, then $\epsilon^{\partial}(+\gamma)$.

Definition 3.2. Given an order-type $\epsilon$, the signed generation tree $* \varphi$, with $* \in\{-,+\}$, of an $\mathcal{L}$-sentence $\varphi\left(p_{1}, \ldots p_{n}\right)$ is $\epsilon$-recursive if every $\epsilon$-critical branch is $\epsilon$-good. Such a signed generation is non-trivially $\epsilon$-recursive if contains at least one $\epsilon$-critical branch.

An $\mathcal{L}$-inequality $\varphi \leq \psi$ is $\epsilon$-recursive if the signed generation trees $+\varphi$ and $-\psi$ are both $\epsilon$-recursive. An $\mathcal{L}$-inequality $\varphi \leq \psi$ is recursive if it is $\epsilon$-recursive for some order-type $\epsilon$.

The signed generation tree $* \varphi$, with $* \in\{-,+\}$, is $\epsilon$-PIA if it is $\epsilon$-recursive and all $\epsilon$-critical branches consist only of PIA-nodes. Such a signed generation is non-trivially $\epsilon$-PIA if contains at least one $\epsilon$-critical branch. 
Example 3.3. Consider the inequality

$$
\diamond \mu X .[(p \vee X) \vee \sim v Y \cdot[\diamond(X \vee \sim((Y \wedge p) \wedge \mu Z . \sim(\square p \wedge \neg Z))) \rightarrow \diamond \square p]] \leq \diamond \square p .
$$

This is $\epsilon$-recursive with $\epsilon_{p}=1$. Indeed, in the positive generation tree of the left-hand side, there are two critical branches, respectively corresponding to the first and third occurrences of $p$ in the formula, counting from the left. The branch leading from the first is $+p,+\vee,+\vee,+\mu X,+\diamond$, and partitioning this as $P_{1}=\varnothing, P_{2}=+\vee,+\vee,+\mu X$, and $P_{3}=+\diamond$, satisfies the requirements of definition 3.2. The branch leading from the third is

$$
+p, \underbrace{+\square,+\wedge,-\sim,-\mu Z}_{P_{1}}, \underbrace{-\wedge,+\sim,+\vee,+\diamond,-\rightarrow,-v Y,+\sim,+\vee,+\mu X}_{P_{2}}, \underbrace{+\diamond}_{P_{3}}
$$

and partitioning it as indicated satisfies the requirements of definition 3.2 . In particular, there are no SRR nodes, and the only occurring SLR node is $-\rightarrow$, which clearly satisfies condition 3(a) of the definition.

Proposition 3.4. When restricted to the classical semantics (boolean algebras with operators or Kripke frames) the class of recursive $\mu$-inequalities strictly contains both the regular formulas of [6] and the Sahlqvist mu-formulas of [8].

\section{Alba succeeds of all recursive $\mu$-inequalities}

Theorem 4.1. ALBA succeeds on all recursive $\mu$-inequalities. Hence every recursive $\mu$-inequality has a FO+LFP correspondent on intuitionistic modal Kripke frame.

Example 4.2. ALBA succeeds on the inequality

$$
\diamond \mu X .[(p \vee X) \vee \sim \nu Y .[\diamond(X \vee \sim((Y \wedge p) \wedge \mu Z . \sim(\square p \wedge \neg Z))) \rightarrow \diamond \square \square p]] \leq \diamond \square p .
$$

which, as discussed in example 3.3 is $\epsilon$-recursive with $\epsilon_{p}=1$.

\section{References}

[1] W Conradie, Y Fomatati, A Palmigiano, and S Sourabh. Algorithmic correspondence for intuitionistic modal mu-calculus. 2013. Journal version, submitted.

[2] W Conradie, Y Fomatati, A Palmigiano, and S Sourabh. Algorithmic correspondence for intuitionistic modal mu-calculus, part 1. 2013. Abstract submitted to TACL2013.

[3] Willem Conradie, Silvio Ghilardi, and Alessandra Palmigiano. Unified correspondence. In Logical/Informational Dynamics, in honor of Johan van Benthem, A. Baltag and S. Smets, eds., forthcoming volume of Trends in Logic: Outstanding Contributions. Springer, 2012.

[4] Willem Conradie and Alessandra Palmigiano. Algorithmic correspondence and canonicity for distributive modal logic. Annals of Pure and Applied Logic, 163(3):338 - 376, 2012.

[5] Willem Conradie, Alessandra Palmigiano, and Sumit Sourabh. Algebraic modal correspondence: Sahlqvist and beyond. Submitted.

[6] V. Goranko and D. Vakarelov. Elementary canonical formulae: Extending Sahlqvist theorem. Annals of Pure and Applied Logic, 141(1-2):180-217, 2006.

[7] Dexter Kozen. Results on the propositional mu-calculus. Theor. Comput. Sci., 27:333-354, 1983.

[8] Johan van Benthem, Nick Bezhanishvili, and Ian M. Hodkinson. Sahlqvist correspondence for modal mucalculus. Studia Logica, 100(1-2):31-60, 2012. 